\title{
Plataforma SAM: a gamificação e a colaboração em uma plataforma de aprendizagem para o ensino da matemática em crianças portadoras de Síndrome de Down
}

\author{
Antonio Victor Alencar Lundgren ${ }^{1}$, Zildomar Carlos Félix ${ }^{2,3}$ \\ ${ }^{1}$ Programa de Pós-Graduação em Engranharia da Computação (PPGEC) \\ Universidade de Pernambuco - POLI - UPE \\ ${ }^{2}$ Laboratório de Estudos em Informática Aplicada (LEIA) \\ Universidade Federal Rural de Pernambuco - UAST/UFRPE \\ ${ }^{3}$ Laboratório de Tecnologias para o Ensino Virtual e Estatística (LabTEVE) \\ Universidade Federal da Paraíba-UFPB \\ aval@ecomp.poli.br, zildomarf@gmail.com
}

\begin{abstract}
This article presents the Plataforma SAM, a web-based, collaborative and gamified learning platform focused on teaching mathematics to youngsters and children with Down 's syndrome, detailing the learning platform development and validation process, which presented excellent results in its user's content retention.
\end{abstract}

Resumo. Este artigo apresenta a Plataforma SAM, uma plataforma de aprendizado web, gamificada e colaborativa voltada ao ensino da matemática para jovens e crianças portadoras de Síndrome de Down, detalhando seu desenvolvimento e processo de validação, o qual apresentou ótimos resultados na retenção de conteúdo de seus usuários.

\section{Introdução}

A Síndrome de Down (SD) é caraterizada por Tempski et al (2016) como um desequilíbrio da constituição cromossômica, onde o par de cromossomos 21 apresenta um cromossomo extra, totalizando 47 cromossomos em comparação aos 46 dos indivíduos com desenvolvimento típico.

De acordo com Breslin et al (2014), a SD é a causa genética mais comum para deficiência intelectual (DI). Tal DI é acentuada quando se trata do aprendizado de conceitos lógico-aritméticos, em comparação ao aprendizado da leitura e/ou escrita (Ahmed, Muddin \& Shafie, 2014). Porém é importante, como explica Carmo (2012), salientar que, sim, tais indivíduos capazes de aprender matemática.

Considerando a importância das habilidades lógico-aritméticas básicas para a realização de atividades comuns, como por exemplo, identificar as horas em um relógio, se torna evidente a necessidade das mesmas por parte de portadores de SD. Portanto foi proposto em 2015 (Lundgren et al, 2015) uma plataforma de aprendizagem gamificada e colaborativa para auxiliar o desenvolvimento das habilidades pré e aritméticas de crianças portadores de SD, a qual foi denominada Plataforma SAM, sigla para Sistema de Auxílio à Matemática. $O$ trabalho aqui presente relata $o$ processo de conceituação, desenvolvimento e validação da Plataforma. 
Este artigo está organizado da seguinte forma: a seção 2 que descreve o referencial teórico importante para o desenvolvimento deste trabalho. A seção 3 mostra os trabalhos relacionados. Já a seção 4 descreve a metodologia utilizada, e na seção 5 mostra a Plataforma SAM e suas funcionalidades. A seção 6 discute a validação e análise dos resultados. Por último, na seção 7 são apresentadas as considerações finais.

\section{Referencial Teórico}

A seguir serão discutidas as áreas relevantes para a criação da ferramenta SAM.

\subsection{Ensino da Matemática para Portadores de Síndrome de Down}

Apesar das dificuldades encontradas por crianças portadoras de SD no aprendizado da matemática, não existem modificações necessárias no conteúdo passado, em relação ao transmitido para crianças sem SD. As dificuldades matemáticas natas apresentadas por crianças portadoras de SD podem ser minimizadas que o conteúdo é apresentado, já que os conceitos lógico-matemáticos estão relacionados a fatores culturais (Bissoto, 2005).

Carmo (2012) propõe um currículo educacional matemático para crianças portadoras de DI, o qual engloba princípios para a educação eficaz, tendo esses princípios apoiados por outros trabalhos (Buckley, 2007; Gomes, 2011) e se mostrando efetivo no ensino para crianças Down. As unidades encontradas no currículo são: habilidades pré-aritméticas, conceito de número, produção de sequências numéricas, produção de conjuntos e subconjuntos, contagem, estimativas aproximadas, soma, subtração, multiplicação e divisão.

Groenwald et al (2010) acredita que as metodologias, bem como as ferramentas tecnológicas utilizadas na educação inclusiva para portadores de DI, especificamente softwares, devem levar em consideração a possiblidade de interação entre o educando e a máquina, considerar as características cognitivas de cada aluno, seus conhecimentos e experiências prévias e suas dificuldades.

Essa preocupação com as peculiaridades dos estudantes pode ser alcançada, segundo Groenwald et al (2010), pelo uso da computação, dado seus benefícios reconhecidos na integração e sua capacidade de compensar situações desfavoráveis de indivíduos.

\subsection{Gamificação na Educação}

Gamificação é definido por Kapp (2012) como o uso de mecânicas e pensamento comumente encontrado em videogames para a criação de engajamento e motivação, em um contexto fora dos videogames. Zichermann \& Cunningham (2011), compararam características comumente encontradas em videogames com estudos da psicologia, como o reforço de Pavlov e Skinner, os associando e descobrindo como tais características podem ser aproveitadas no processo da gamificação.

Em relação ao uso da gamificação para a educação, diversos são os estudos que mostram melhorias em aspectos do aprendizado, como motivação, engajamento e rendimento. (Neto, da Silva \& Bittencourt, 2015; Hamari, Koivisto \& Sarsa, 2014). Quando levado em consideração a maneira como a atenção das pessoas vem mudando nos últimos anos (Kapp, 2012), torna-se evidente a necessidade de modificações no ensino, a necessidade de maior envolvimento e motivação. 
VI Congresso Brasileiro de Informática na Educação (CBIE 2017)

Anais do XXVIII Simpósio Brasileiro de Informática na Educação (SBIE 2017)

\section{Trabalhos Relacionados}

Foi observada uma grande escassez de trabalhos que abordam a temática. Dois trabalhos foram considerados relevantes para o desenvolvimento deste: o programa Somar e a aplicação móvel MathDS.

O programa Somar (Thomaz \& Moureira, 2014) é um projeto da Universidade de Brasília (UnB), desenvolvido para auxiliar o ensino da matemática básica para jovensadultos portadores de SD, o qual aborda o conceito de número, soma, subtração, tempo e dinheiro. É um software desenvolvido em flash, portanto é multiplataforma.

A aplicação MathDS (Ahmad, Muddin \& Shafie, 2010), da Universiti Teknologi Petronas (UTP), é uma aplicação móvel disponível para a plataforma Andriod. O MathDS trata apenas do conceito de número. O Quadro 1 apresenta uma comparação das características encontradas no Somar e no MathDS com as características encontradas na Plataforma SAM.

Quadro 1. Comparação das características entre os trabalhos relacionados.

\begin{tabular}{|c|l|l|l|l|}
\hline$N^{\circ}$ & \multicolumn{1}{|c|}{ Característica } & Program Somar & MathDS & S.A.M. \\
\hline $\mathbf{1}$ & Utiliza imagens & Sim & Sim & Sim \\
\hline $\mathbf{2}$ & Utiliza efeitos sonoros & Sim & Sim & Sim \\
\hline $\mathbf{3}$ & Utiliza vídeo & Sim & Não & Não \\
\hline $\mathbf{4}$ & Faz uso de interface amigável & Não & Sim & Sim \\
\hline $\mathbf{6}$ & Aborda o conceito de contagem & Não & Não & Sim \\
\hline $\mathbf{7}$ & Aborda o conceito de número & Sim & Sim & Sim \\
\hline $\mathbf{8}$ & Aborda a soma & Sim & Não & Sim \\
\hline $\mathbf{9}$ & Aborda a substração & Sim & Não & Não \\
\hline $\mathbf{1 0}$ & Faz uso da gamificação & Não & Não & Sim \\
\hline $\mathbf{1 1}$ & Multiplataforma & Sim & Não & Sim \\
\hline $\mathbf{1 2}$ & Colaborativo & Não & Não & Sim \\
\hline
\end{tabular}

\section{Considerações Metodológicas}

O desenvolvimento deste trabalho foi dividido em quatro fases, visando possíveis modificações futuras ao projeto. Na primeira fase foi realizada uma pesquisa para identificar carências na educação inclusiva e áreas consideradas relevantes para o projeto, tal pesquisa foi realizada em diversas bases científica. Ao fim da primeira iteração desta fase foi possível observar a escassez existente em ferramentas brasileiras cujo objetivam auxiliar o ensino da matemática para crianças portadoras de SD.

A fase 2 envolveu o esboço das características e funcionalidades desejadas para a plataforma, incluindo seu nome. Foram utilizados mapas mentais, storyboards e diagramas de caso de uso para criar os esboços iniciais da plataforma. Nessa fase também foi planejado o roteiro que as atividades seguiriam, a abordagem às dificuldades apresentadas por crianças Down e como a colaboração entre professores funcionaria.

Durante a fase 3 foi realizado o desenvolvimento da Plataforma, de acordo com os requisitos encontrados durante a fase 2. O software foi desenvolvido para a plataforma web, dada as vantagens que esse meio apresenta para a educação, como por exemplo, a fácil modificação e/ou adição de conteúdo. Já a última fase do ciclo de desenvolvimento do projeto foi a validação, tal fase é melhor detalhada na Seção 6.

\section{A plataforma SAM}

Durante o planejamento da Plataforma SAM, se tornou prioridade que a ferramenta criada facilitasse a obtenção de conteúdo por portadores de SD, mas que também os 
motivasse a querer aprender e de permanecer utilizando a plataforma. Para a melhor associação e relação entre os estudantes e a plataforma e uma maior sensação de conforto, foi criado um mascote, o qual foi chamado Sanzinho, uma figura feliz, criada como uma caricatura animada do numeral cinco.

Além disso, idealizou-se a utilização de um único ambiente familiar ao estudante para o estudo de múltiplos conteúdos da matemática, portanto foi decidido que a plataforma criada possibilitaria a criação e compartilhamento de novas atividades e módulos de conteúdo por parte dos professores.

Para a transmissão do conteúdo aos estudantes foi desenvolvida uma hierarquia de conteúdos. A hierarquia é composta por: 1) Aventuras, que podem ser entendidas como estruturas curriculares que serão trabalhadas com os estudantes, aventuras podem ser criadas e compartilhadas livremente entre professores; Aventuras são compostas por 2) módulos, os quais são conjuntos de atividades que abordam um único assunto, módulos devem ser completados em ordem sequencial, ou seja, para realizar atividades de um módulo, é necessário completar os módulos predecessores; 3) Atividades são listas de exercícios formadas por questões que devem ser completadas sequencialmente; e 4) Questões, as quais podem ser de múltipla escolha ou abertas.

\subsection{Requisitos da Plataforma}

Em relação aos requisitos da plataforma, serão destacados aqui os requisitos educacionais (Quadro 2), os quais visam minimizar os impactos das deficiências das crianças e jovens portadores de SD.

\section{Quadro 2 Requisitos Educacionais da Plataforma SAM}

\begin{tabular}{|r|l|}
\hline No & Requisito Educacional \\
\hline 1 & As questões devem possuir dicas que facilitem a realização pelo usuário, motivando o mesmo \\
\hline 2 & $\begin{array}{l}\text { O tamanho da fonte e dos botões deve ser grande, buscando facilitar o entendimento da questão por parte do } \\
\text { estudante, dado o quão comum são problemas de vista em portadores de SD }\end{array}$ \\
\hline 3 & Textos escritos relevantes para a realização de exercícios devem ser lidos por meio de um sintetizador de voz \\
\hline 4 & $\begin{array}{l}\text { Imagens utilizadas nas atividades da aventura padrão da plataforma devem representar objetos que os usuários } \\
\text { reconheçam em seu dia-a-dia }\end{array}$ \\
\hline 5 & $\begin{array}{l}\text { Objetos que possam transmitir duplicidade, como tesouras ou óculos, não foram utilizados, evitando confusões } \\
\text { durante contagens }\end{array}$ \\
\hline 6 & Ao acertar uma questão ou uma atividade um som de sucesso deve ser reproduzido \\
\hline 7 & A interface deve ser limpa e clara, evitando dispersar a atenção do usuário durante a realização de atividades \\
\hline
\end{tabular}

\subsection{Funcionalidades}

As funcionalidades encontradas na Plataforma SAM podem ser divididas pelo perfil de usuário: Estudante e Professor.

\subsubsection{Perfil do Estudante}

Caso o usuário seja um estudante, após a autenticação ele é levado à tela de aventura, essa tela apresenta ao estudante "A Primeira Aventura" ou outro currículo de atividades designado pelo seu professor. Aventuras são representadas por meio de um jogo de tabuleiro, onde o estudante avança as casas completando atividades em uma determinada ordem, objetivando alcançar a última casa do tabuleiro. A página de aventura também apresenta um resumo do progresso do estudante, no que condiz às mecânicas de gamificação, apresentando o nível do seu personagem, a classe atualmente ativa e sua representação gráfica e a quantidade de pontos obtidos realizando atividades. 
Clicando em uma das casas do tabuleiro da aventura o estudante é levado à Atividade relacionada à casa, onde questões são apresentadas em forma de batalha, o personagem do estudante batalhando com um monstro. Ao acertar Questões corações do monstro são retirados, enquanto do personagem do usuário são removidos ao errar questões, a atividade termina quando um dos lados perde todos os corações. Questão e alternativas são lidas para o estudante pelo sintetizador de voz e sons e animações são usados para representar acertos e erros, sendo esses sons e animações simples o suficiente para não distrair o estudante da atividade. Ao fim de uma atividade um som ou mensagem de reforço positivo é reproduzido.

Figura 1. Tela de aventura, tela inicial para o perfil de estudante.

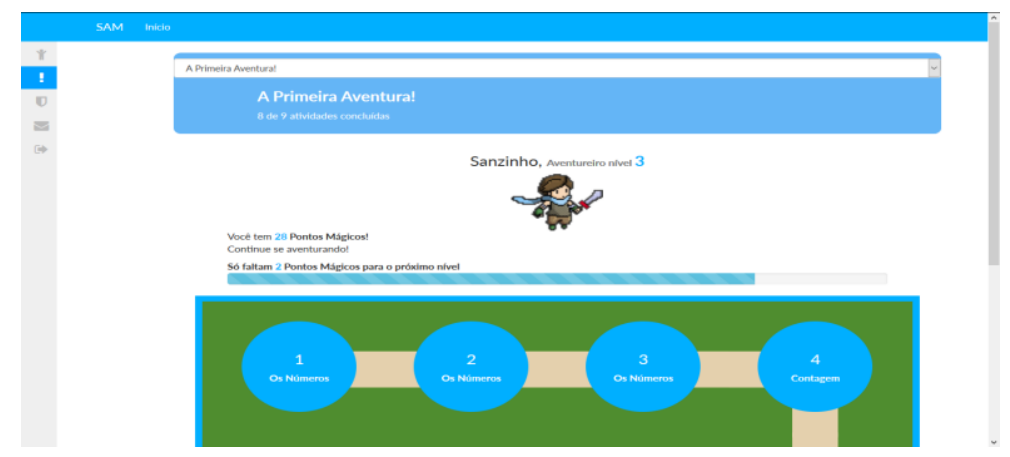

Técnicas de gamificação foram utilizadas no perfil do estudante por meio de pontos, níveis e classes, os quais são recompensados pelos esforços do estudante na plataforma. Classes são relacionadas a quantidades de atividades realizadas, nível alcançado e tempo realizando atividades na plataforma, oferecendo um senso de progresso, dado que as classes se tornam visualmente mais poderosas conforme são liberadas.

Informações sobre o progresso e desempenho do estudante podem ser melhor acompanhados na página de perfil do estudante. Essa página apresenta as maiores dificuldades do estudante, aventura, módulo e atividade atualmente sendo realizadas, além de fornecer o nome do professor responsável pelo estudante.

\subsubsection{Perfil do Professor}

Caso o usuário autenticado seja um professor, sua tela inicial representa a tela da turma. A tela da turma é onde o professor pode gerenciar e acompanhar os estudantes que estão sob sua responsabilidade. A tela resume informações do estudante em cartões, que quando clicados levam ao perfil do estudante, permitindo o melhor acompanhamento.

Figura 2. Tela da biblioteca do professor.

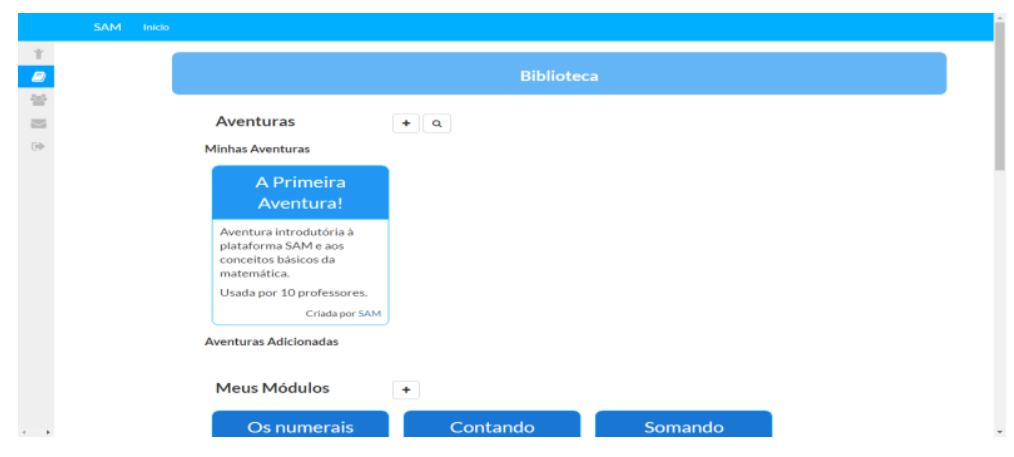


O professor pode também gerenciar, adicionar e criar novo conteúdo para a plataforma, na tela de biblioteca. A tela (Figura 2) é dividida pela hierarquia de conteúdo mencionada anteriormente e apresenta cartões que representam o conteúdo presente na biblioteca do professor. O professor possui as opções de buscar aventuras criadas por outros professores ou criar novo conteúdo de determinado nível da hierarquia.

Aventuras e módulos possuem um nome e uma descrição de seu conteúdo. Para a criação de uma nova aventura é necessário módulos, podendo o professor criar novos ou buscar módulos já criados. Módulos necessitam de um mínimo de três atividades, as quais podem adicionadas das já criadas na plataforma ou criadas novas.

Por fim, as atividades não possuem nome, recebendo o nome do tema que as mesmas tratam, mas possuem uma descrição que resume o que a atividade trata. Questões possuem um enunciado, uma dica, uma imagem e, caso sejam de múltipla escolha, até quatro alternativas.

\subsection{A Primeira Aventura}

A Primeira Aventura é o nome dado ao currículo base da Plataforma SAM, criado para transmitir conhecimentos sobre o conceito de número, contagem e adição básica, além de servir também como exemplo das capacidades de criação de conteúdo na plataforma.

A aventura está dividida em três módulos: Os Números, Contagem e Adição. Cada um dos módulos possui três atividades, totalizando nove atividades. As atividades planejadas para essa aventura levaram em consideração o currículo de Carmo (2012), mencionado anteriormente. As atividades possuem a preocupação de assimilar os conceitos com atividades e objetos do dia-a-dia do estudante.

O primeiro módulo, "Os Números", trata dos numerais de um a nove, onde cada atividade trabalha três desses numerais. As atividades buscam não só apresentar os numerais em suas formas extensas e cardinais, mas também realizar a assimilação entre essas formas de escrita, quantidades de objetos e a pronuncia por meio do uso do sintetizador de voz. A Figura 3 demonstra uma das atividades do primeiro módulo.

Figura 3. Atividade sobre o conceito de números.

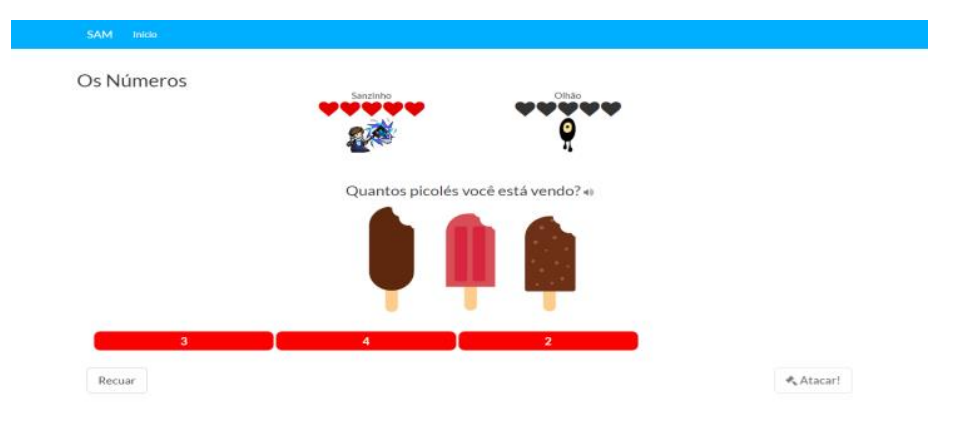

O segundo módulo da aventura, denominado Contagem, aborda sequências numéricas (Figura 4a), contagem de conjuntos de objetos e solução de situações onde é necessária a contagem para saber a resposta.

O terceiro módulo da Primeira Aventura trata a adição, bem como a identificação do símbolo de adição. As questões nesse módulo abordam a identificação do símbolo de adição em uma soma simples, a junção entre dois conjuntos de objeto e a 
VI Congresso Brasileiro de Informática na Educação (CBIE 2017)

Anais do XXVIII Simpósio Brasileiro de Informática na Educação (SBIE 2017)

soma entre dois numerais menores que nove, dado que esses são os numerais trabalhados no módulo Os Números. A Figura 4(b) representa uma atividade sobre adição.

Figura 4. Atividade sobre contagem e Adição.

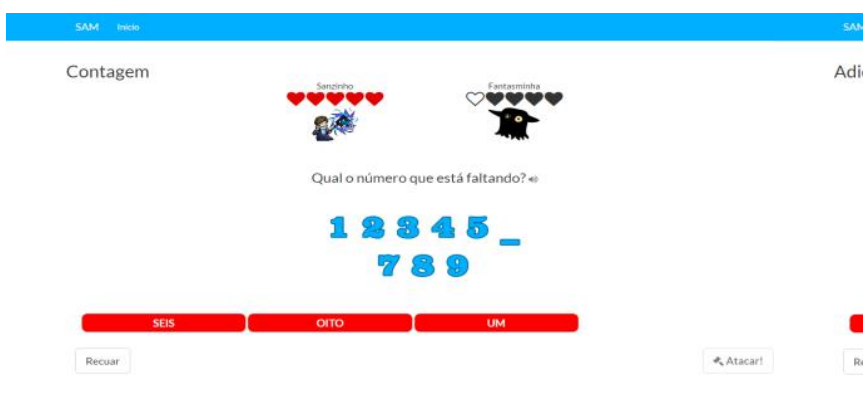

(a)

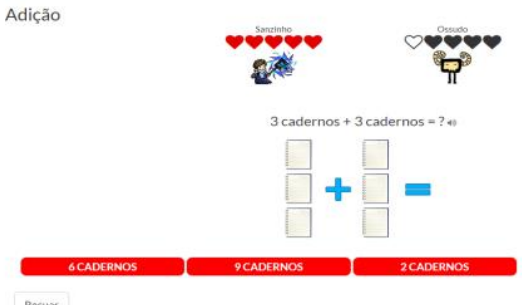

(b)

\section{Validação}

O processo de validação ocorreu por meio da aplicação da plataforma em uma situação de uso real. A pesquisa de validação ocorreu com frequentadores da Associação de Pais e Amigos dos Excepcionais (APAE) e em uma clínica de psicopedagogia, mediante autorização dos pais ou responsáveis dos participantes por meio de assinatura em um termo de consentimento.

Para a pesquisa foram selecionados quatro participantes portadores de SD, entre 14 e 33 anos, dos quais três não frequentam o sistema de ensino regular e são analfabetos. Para preservar suas identidades os participantes foram denominados P1, P2, P3 e P4. Suas idades são respectivamente: 23, 17, 33 e 14 anos. O processo de validação pode ser dividido em três etapas: 1) pré-teste; 2) aplicação da validação; 3) pós-teste.

Antes do início do processo de validação em si, foi realizada uma apresentação da plataforma e treinamento para os profissionais das instituições.

\subsection{Aplicação da Validação}

O primeiro passo da validação constituiu um pré-teste, onde o objetivo foi de identificar os conhecimentos prévios dos participantes. Para tal foram desenvolvidos 28 cartões, os quais simulam questões da plataforma. O processo envolvia apresentar os cartões aos participantes, realizar a pergunta do cartão apresentado e receber uma resposta. O pré-teste foi realizado individualmente com os participantes.

A aplicação da plataforma ocorreu em três utilizações, ao longo de duas semanas. Os participantes utilizaram "A Primeira Aventura" da plataforma com o objetivo de percorrer as nove atividades que esta aventura apresenta.

Após a aplicação da plataforma foi realizado um pós-teste, o qual é a repetição do mesmo processo do pré-teste, comparando os resultados de ambos o pré e o pósteste foi possível identificar a retenção de conhecimento derivada da utilização da plataforma. Por fim foram realizados questionários, com ambos profissionais e estudantes, a respeito da opinião e experiência deles com a plataforma SAM.

\subsection{Análise dos Resultados}


VI Congresso Brasileiro de Informática na Educação (CBIE 2017)

Anais do XXVIII Simpósio Brasileiro de Informática na Educação (SBIE 2017)

Após o pré-teste, os resultados dos participantes foram analisados. O Quadro 3 mostra a quantidade de acertos no pré-teste com cada participante, do total de vinte e oito (28) perguntas realizadas.

Quadro 3 Acertos dos participantes no Pré-teste

\begin{tabular}{|l|r|r|r|r|}
\hline \multirow{2}{*}{ Módulo } & \multicolumn{4}{|c|}{ Participante } \\
\cline { 2 - 5 } & P1 & P2 & P3 & P4 \\
\hline Conceito de Número & 1 & 0 & 1 & 9 \\
\hline Contagem & 2 & 2 & 2 & 9 \\
\hline Soma & 1 & 1 & 2 & 8 \\
\hline Total & 4 & $\mathbf{3}$ & 5 & $\mathbf{2 6}$ \\
\hline
\end{tabular}

A aplicação da plataforma com os participantes ocorreu individualmente, onde cada um dos participantes era levado à sala de informática da instituição e com o auxílio de um profissional utilizavam a plataforma. O Quadro 4 apresenta um resumo do desempenho dos participante na utilização da plataforma, apresentando a quantidade de sessões realizadas, quantidade de atividades concluídas na "Primeira Aventura" e maior dificuldade de acordo com o acompanhamento da própria Plataforma SAM.

Quadro 4 Resumo da aplicação da plataforma com os participantes

\begin{tabular}{|l|r|r|r|r|}
\cline { 2 - 5 } \multicolumn{1}{c|}{} & P1 & P2 & P3 & P4 \\
\hline Sessões de uso realizadas & 3 & 1 & 3 & 3 \\
\hline Atividades finalizadas & 3 & 0 & 3 & 9 \\
\hline Maior dificuldade & Contagem & Os Números & Contagem & Soma \\
\hline
\end{tabular}

É importante salientar que, com exceção do participante 2, todos os outros demonstraram animação e motivação ao utilizar a plataforma, comemorando seus acertos, quando a animação ocorria e quando derrotavam o monstro, e suas conquistas ao liberar novas classes. Ao fim da aplicação da plataforma, outra visita foi marcada para a realização do pós-teste. Foi verificada a retenção de conhecimento - nos resultados de todos os participantes que realizaram o pós-teste, como mostra a Figura 5. Os participantes P1, P2 e P3 apresentaram uma porcentagem de acertos de, respectivamente, $29 \%$, 36\% e 100\%, em comparação aos $14 \%$, 18\% e 93\% de acerto no pré-teste.

Figura 5. Comparação dos resultados do pré e pós-teste dos participantes.

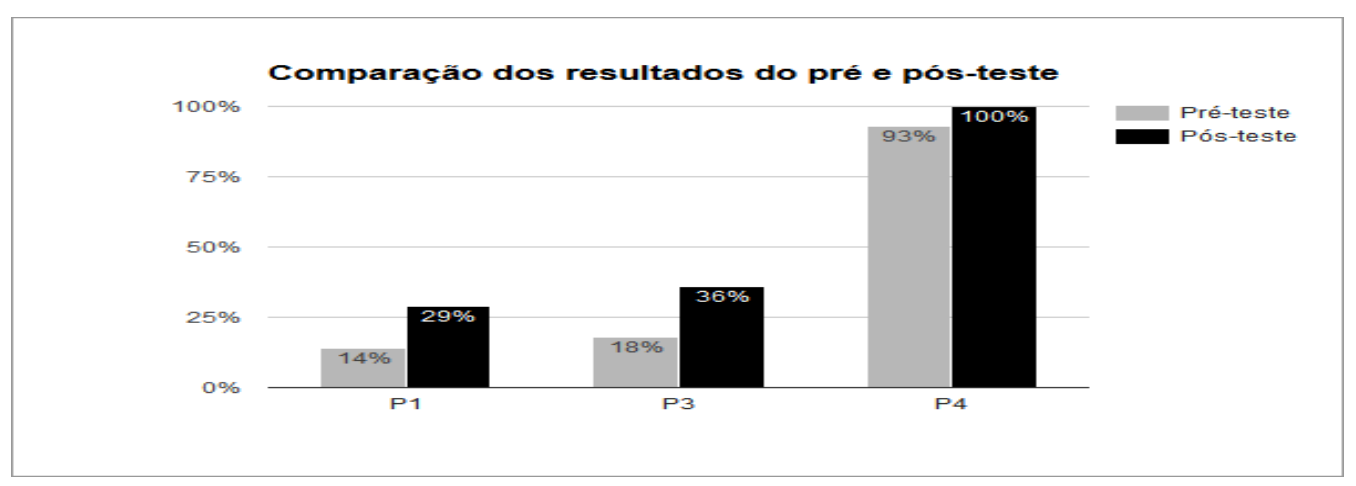

Por fim, foi realizado um questionário com ambos os participantes e profissionais para obter as opiniões a respeito da plataforma. Aos participantes foi questionado: a) a respeito da dificuldade das atividades, $66 \%$ responderam que acharam as questões difíceis; b) a respeito do uso de voz na plataforma, onde $66 \%$ respondeu que gostaram do uso de voz; c) a opinião dos participantes a respeito das batalhas nas atividades, $100 \%$ responderam que gostaram das batalhas; d) se os participantes gostaram das classes na plataforma, $100 \%$ respondeu que gostou das classes; e f) foi 
VI Congresso Brasileiro de Informática na Educação (CBIE 2017)

Anais do XXVIII Simpósio Brasileiro de Informática na Educação (SBIE 2017)

questionado se os participantes gostaram de usar a plataforma e se gostariam de usar novamente, onde $100 \%$ respondeu que sim.

Já aos profissionais foi passado um questionário com dez questões, as perguntas variavam desde experiência com softwares educacionais e com a Plataforma SAM, até visão para o futuro da plataforma. O questionário foi realizado com dois profissionais, um de cada uma das instituições onde a pesquisa foi realizada. A análise dos resultados deste questionário está visível no Quadro 5.

Quadro 5 Resultados do questionário realizado com os profissionais

\begin{tabular}{|c|c|c|}
\hline Pergunta & Sim & Não \\
\hline Já fez uso de softwares educacionais & $50 \%$ & $50 \%$ \\
\hline Acredita nos benefícios da ludificação na educação & $100 \%$ & $0 \%$ \\
\hline Acha as atividades da plataforma são adequadas para a transmissão do conteúdo & $100 \%$ & $0 \%$ \\
\hline Acha que a as atividades poderiam possuir mais exercícios & $50 \%$ & $50 \%$ \\
\hline Acha a dificuldade das atividades da plataforma adequadas & $50 \%$ & $50 \%$ \\
\hline Acha que as atividades da plataforma poderiam ser mais difíceis & $50 \%$ & $50 \%$ \\
\hline Acredita na importância da criação e compartilhamento de counteúdo na plataforma & $100 \%$ & $0 \%$ \\
\hline Classifica a criação e compartilhamento de conteúdo da plataforma como excelente & $100 \%$ & $0 \%$ \\
\hline Classifica a plataforma como excelente & $100 \%$ & $0 \%$ \\
\hline Faria uso da plataforma em sua sala de aula & $100 \%$ & $0 \%$ \\
\hline Acredita na expansão da plataforma para portadores de outras deficiências intelectuais & $100 \%$ & $0 \%$ \\
\hline
\end{tabular}

\section{Conclusão}

A plataforma SAM possui o potencial e facilidade de ser utilizada em grande escala devido a seu ambiente de execução, além de poder trazer também benefícios reais aos seus usuários, dado as características da plataforma e os resultados obtidos durante a validação da plataforma, com resultados apontando o aprimoramento médio nas capacidades matemáticas dos participantes de 36,24\%.

Entre as contribuições deste trabalho estão os seguintes artefatos: 1) a identificação do processo de aprendizagem da matemática em crianças e jovens portadores de SD, bem como estratégias para a abordagem desse processo; 2) como mecânicas de videogames, por meio da gamificação, podem ser utilizadas para aprimorar o aprendizado da matemática e sua experiência para crianças portadoras de SD; e 3) o desenvolvimento aplicação web, gamificada e colaborativa capaz de auxiliar no processo de ensino da matemática para portadores de SD.

A maior dificuldade encontrada durante este trabalho foi a pequena quantidade de participantes disponíveis para a validação da plataforma, esta quantidade reduzida de portadores de SD nas instituições onde a pesquisa foi realizada se dá, acredita-se, à reduzida quantidade de habitantes da cidade onde a pesquisa foi realizada. Entre outras dificuldades se encontram as condições estruturais durante a aplicação, as quais apesar de suficientes era, em determinados momentos, não foram favoráveis ao ensino.

Por fim, este trabalho abre o espaço para futuros trabalhos, incluindo a possibilidade de expansão da plataforma para outras deficiências intelectuais, além da SD e possível confirmação da validação em melhores condições.

\section{Referências Bibliográficas}

Ahmad, W. F. W., Muddin, H. N. B. I., \& Shafie, A. (2014). Number skills mobile application for down syndrome children. Computer and Information Sciences (ICCOINS), 2014. International Conference on. IEEE. 
VI Congresso Brasileiro de Informática na Educação (CBIE 2017)

Anais do XXVIII Simpósio Brasileiro de Informática na Educação (SBIE 2017)

Bissoto, M. L. (2006). O desenvolvimento cognitivo e o processo de aprendizagem do portador de Síndrome de Down: Revendo concepções e perspectivas educacionais. SP, Brasil: Ciências \& Cognição. 2005.

Breslin, J., Spanò, G., Bootzin, R., Anand, P., Nadel, L., \& Edgin, J. (2014). Obstructive sleep apnea syndrome and cognition in Down syndrome. Developmental Medicine \& Child Neurology, 56(7), 657-664.

Buckley, S. J. (2007). Teaching numeracy. Down Syndrome Research and Practice.

Carmo, J. S. (2012) Aprendizagem de conceitos matemáticos em pessoas com deficiência intelectual. Revista de Deficiência Intelectual, v. 3. p. 43-48.

Gomes, R. A. O. (2011) Processo de Ensino Aprendizagem da Matemática Para Alunos Portadores de Síndrome de Down. Trabalho de conclusão de Curso.

Groenwald, C. L. O., Seibert, T. E., Moreno, L., Muñoz, V., da Hora, G. S., de Matos, A. C.,\& Cafeseiro, J. S. (2011). Eixos convergentes na aprendizagem matemática de alunos com Síndrome de Down. Rev. Eletr. de Educação Matemática, 5(1), 25-37.

Hamari, J., Koivisto, J., \& Sarsa, H. (2014). Does gamification work?--a literature review of empirical studies on gamification. In System Sciences (HICSS), 2014 47th Hawaii International Conference. p. 3025-303. IEEE.

Kapp, K. M. (2012) The gamification of learning and instruction: game-based methods and strategies for training and education. John Wiley \& Sons.

Lundgren, A. V., Santos, D. G. D. S., Luna, F. D. M., Felix, Z. C., \& de Almeida Rodrigues, I.(2015) SAM: Uma plataforma gamificada de ensino a matemática voltada a crianças com Síndrome de Down. In Simp. de Ex. ${ }^{a}$ em Gestão e Tecnol. (XII SEGeT).

Neto, A., da Silva, A. P., \& Bittencourt, I. I. (2015).Uma análise do impacto da utilização de técnicas de gamificação como estratégia didática no aprendizado dos alunos.Anais do Simp. bras. de Inform. na Educ. V26, No. 1. p. 667.

Tempski, P. Z., Miyahara, K. L., Almeida, M. D., de Oliveira, R. B., Oyakawa, A., \& Battistella, L. R. (2016). Protocolo de cuidado à saúde da pessoa com Síndrome de Down-IMREA/HCFMUSP. Acta fisiátrica, 18(4), 175-186.

Thomaz, L.S.S.,\& Moreira, T.E.G.(2014).Somar: ferramenta educacional de apoio ao ensino da matemática aplicada ao cotidiano de jovens e adultos com deficiência intelectual.

Zichermann, G., Cunningham, C. (2011) Gamification by Design: Implementing Game Mechanics in Web and Mobile Apps. O'Reilly Media, Inc. 